All authors: University of California, San Francisco Helen Diller Family Comprehensive Cancer Center, University of California, San Francisco, CA.

Submitted February 7, 2011; accepted June 7, 2011; published online ahead of print at www.jco.org on August 15, 2011

Supported by an unrestricted educational gift from Abbott Laboratories (Chicago, IL), by the National Institutes of Health/National Cancer Institute grant No. 5RC1CA146596, and by the Agency for Healthcare Research and Quality grant No. 1U01CA88160.

S.P. and M.R.C. both contributed equally to this work.

Authors' disclosures of potential conflicts of interest and author contributions are found at the end of this article.

Corresponding author: Matthew R. Cooperberg, MD, MPH, University of California, San Francisco, Department of Urology, 1600 Divisadero St, Box 1695, San Francisco, CA 94143-1695, mcooperberg@urology.ucsf.edu.

(C) 2011 by American Society of Clinica Oncology

0732-183X/11/2926-3510/\$20.00

DOI: 10.1200/JCO.2011.35.1494

\title{
Androgen Deprivation Therapy and Cardiovascular Risk
}

Sanoj Punnen, Matthew R. Cooperberg, Natalia Sadetsky, and Peter R. Carroll

See accompanying editorial on page 3500; listen to the podcast by Dr D'Amico at www.jco.org/podcast

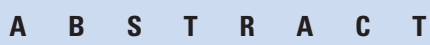

\section{Purpose}

The potential association between androgen deprivation therapy (ADT) and cardiovascular mortality (CVM) remains controversial. This study assessed mortality outcomes in a large national registry to further elucidate the association between treatment selection and cause of mortality.

\section{Patients and Methods}

A total of 7,248 men in the CaPSURE registry were analyzed. Treatment was categorized as local only, primary ADT monotherapy, local treatment plus ADT, and watchful waiting/active surveillance (WW/AS). Competing hazards survival analysis was performed for prostate cancer-specific mortality (PCSM), CVM, and all-cause mortality. A propensity score-adjusted and a propensitymatched analysis were undertaken to adjust for imbalances in covariates among men receiving various treatments.

\section{Results}

Patients treated with ADT or WW/AS had a higher likelihood of PCSM than those treated with local therapy alone. Patients treated with primary ADT had an almost two-fold greater likelihood of CVM ( $\mathrm{HR}, 1.94 ; 95 \% \mathrm{Cl}, 1.29$ to 2.97$)$ than those treated with local therapy alone; however, patients treated with WW/AS had a greater than two-fold increased risk of CVM (HR, 2.46; 95\% Cl, 1.53 to 3.95). A propensity-matching algorithm in a subset of 1,391 patients was unable to find a significant difference in CVM between those who did or did not receive ADT.

\section{Conclusion}

Patients matched on propensity to receive ADT did not show an association between ADT and CVM. This suggests that potential unmeasured variables affecting treatment selection may confound the relationship between ADT use and cardiovascular risk. However, an association may yet exist, because the propensity score could not include all known risk factors for CVM.

\section{J Clin Oncol 29:3510-3516. (9) 2011 by American Society of Clinical Oncology}

\section{INTRODUCTION}

Prostate cancer is the second most common cancer-related cause of death among men in the United States, ${ }^{1}$ with a mortality rate of 9.9 per $100,000 .^{2}$ For patients with metastatic prostate cancer, androgen deprivation therapy (ADT) is the primary method of treatment. ${ }^{3} \mathrm{ADT}$ is also used in combination with local treatment modalities to achieve better cancer control ${ }^{4,5}$ or as an effective salvage therapy in patients who do not respond to local therapy. ${ }^{3,6}$ As many as $46 \%$ of men with prostate cancer receive ADT at some point during their treatment. ${ }^{7,8}$ Most commonly, ADT is achieved by medical castration with gonadotropin-releasing hormone $(\mathrm{GnRH})$ agonists.

Although ADT provides a means of effective cancer control, studies have raised concerns regard- ing the possible adverse effects of ADT on metabolism and subsequent cardiovascular risk. ${ }^{9-12}$ The mechanisms through which ADT has been suspected to increase cardiovascular risk include increased fat mass, increased low-density lipoprotein and total cholesterol, increased triglycerides, and increased insulin resistance. ${ }^{13-15}$

Despite these findings, the relationship between ADT and cardiovascular mortality (CVM) remains controversial; some studies have identified a statistically significant association, whereas others have not. ${ }^{16-18}$ One hypothesis is that ADT potentiates preexisting cardiac risk factors, making them more apparent during ADT initiation. ${ }^{19}$ However, factors that play a role in treatment selection may also confound the relationship between ADT use and CVM. For instance, men who are selected for primary treatment with ADT are typically older in 
age or worse in overall health than men selected for definitive treatment ${ }^{3}$ and thereby may have an increased risk for cardiovascular death.

To better comprehend the impact of treatment selection on mortality, mortality outcomes by treatment course were analyzed in a large, national, prostate cancer registry. In an attempt to control for various known and unknown factors that may confound the relationship between ADT and CVM, patients who used and did not use ADT were matched by their propensity to receive ADT in a subset of our patient cohort.

\section{PATIENTS AND METHODS}

\section{Patient Population}

The Cancer of the Prostate Strategic Urologic Research Endeavor (CaPSURE) is a national cancer registry of men with biopsy-proven prostate cancer, recruited from 40 predominantly community-based urology practices. Men were recruited, at the time of prostate cancer diagnosis, by participating urologists who reported initial and follow-up data, including primary and secondary ADT use. Information on comorbidities is ascertained via a selfreported questionnaire given to patients at the time of enrollment. Specific comorbidities ascertained included hypertension, heart disease, diabetes, lung disease, kidney disease, blood disease, endocrine disorders, neurologic disorders, and mental health conditions. Patients provide written, informed consent under local and central institutional review board approval. Patients were treated as per their clinicians' standard care and were observed until death or study withdrawal. Details regarding the CaPSURE methodology have been reported previously. ${ }^{20,21}$

Men in the CaPSURE database, diagnosed with prostate cancer between 1995 and 2007 with localized disease (clinical stage T1 to T3a) were analyzed. Primary treatment was defined as local only (surgery or radiation), local and ADT (neoadjuvant, adjuvant, and/or salvage ADT), ADT only, or watchful waiting (WW)/active surveillance (AS; WW/AS). WW and AS were combined, because they could not be differentiated in the CaPSURE database. Exclusion criteria included men who were found to have metastatic disease at diagnosis or missing clinical data with respect to prostate-specific antigen (PSA), stage, and/or Gleason score. Men whose primary treatment was unknown, or who were treated with an antiandrogen only, were also excluded from analysis. Finally, men in the local-plus-ADT arm who were treated with ADT for less than 6 months were excluded, because it was not clear that they received ADT as definitive secondary treatment (neoadju- vant, adjuvant, or salvage). However, a sensitivity analysis was performed that included these patients.

Mortality was reported in CaPSURE by participating clinicians, after which a copy of the death certificate was obtained. The cause of death (ie, prostate cancer-specific mortality [PCSM] $v$ CVM $v$ other-cause mortality) was made by consensus of the study investigators. In general, death was considered to be cancer-specific mortality if prostate cancer was listed as a primary, secondary, or tertiary cause of death and if no other malignancy or $\mathrm{CVM}$ was listed as a higher-order cause. CVM was defined as a death as a result of acute myocardial infarction, sudden cardiac arrest or death, coronary artery disease, cardiac ischemia, or malignant arrhythmia. If the patient was lost to follow-up or the state death certificate was not available, the National Death Index was queried to identify date and cause of death.

\section{Statistical Analysis}

The Kaplan-Meier product-limit method was used to test associations among types of treatment and each mortality end point of interest: overall mortality, PCSM, and CVM. Competing risks regression was used to evaluate association of each mortality end point with type of treatment. ${ }^{22}$ This analysis was adjusted for self-reported cardiovascular disease, hypertension, and diabetes mellitus at baseline, as well as age at diagnosis, PSA, clinical T stage, biopsy, and Gleason grade. Adjusted hazard ratios (HRs) with $95 \%$ CIs were reported. Cancer risk was classified by using the University of California, San Francisco, Cancer of the Prostate Risk Assessment score as described previously, and men were assigned to low, intermediate, and high risk on the basis of Cancer of the Prostate Risk Assessment scores 0 to 2,3 to 5 , and 6 to 10 , respectively. ${ }^{23}$

Propensity score analysis was undertaken in an effort to adjust for potential bias associated with factors related to receiving specific treatments. ${ }^{24}$ For each patient, propensity to receive ADT was determined by using multivariable logistic regression that included the following covariates: type of initial treatment; age, PSA, Gleason score, and T stage at diagnosis; number of positive biopsies; insurance status; ethnicity; education; household income; marital status; specific comorbidities (eg, heart disease, hypertension, and diabetes); and year of diagnosis.

Propensity scores thus generated were expressed as a predicted proportion of patients receiving ADT. For the purposes of this analysis, given low numbers of patients in the highest and lowest quintiles, these quintiles were omitted, and three categories ( 0.1 to $0.3,0.3$ to 0.6 , and $>0.6$, with asymmetric cutoffs as a result of distributional issues) were created and used in multivariate Cox proportional hazard analysis. This is because men in the lowest quintile have an almost zero percent chance of being treated with ADT, whereas men in the highest quintile have an almost $100 \%$ chance of being treated with ADT.

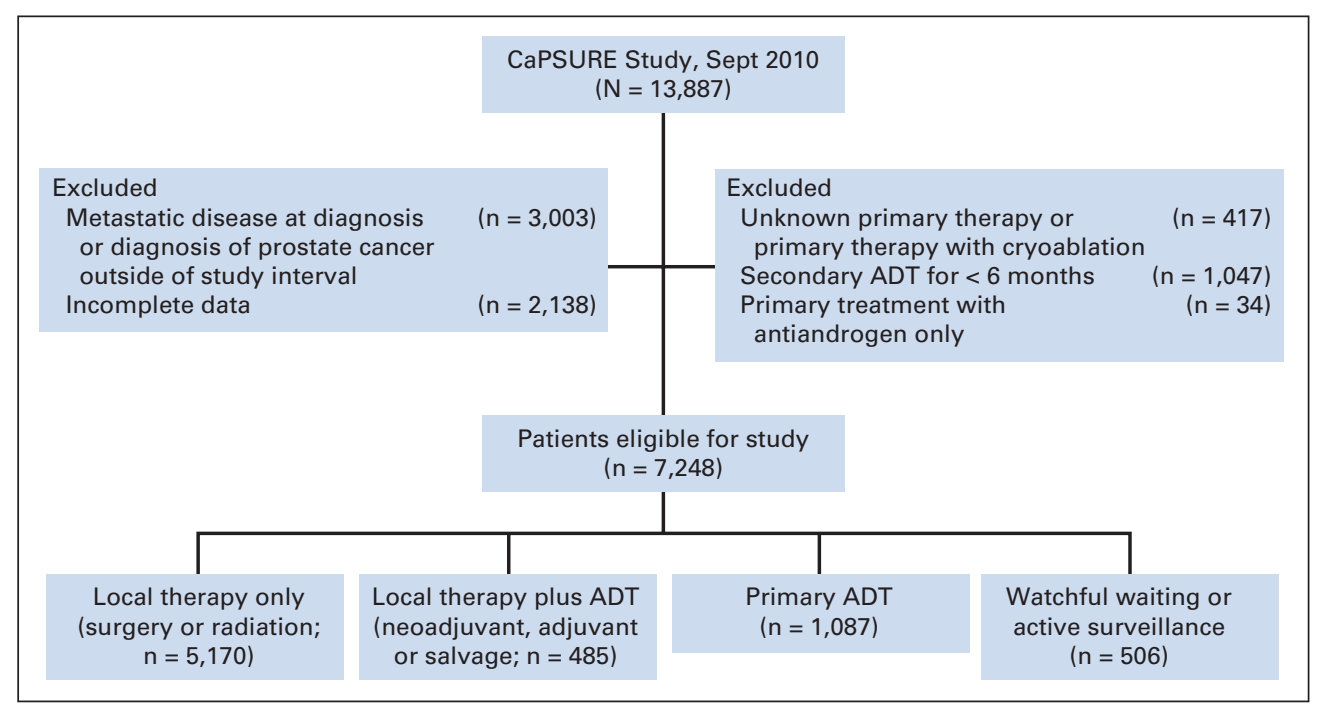

Fig 1. Patient selection and flow chart. ADT, androgen deprivation therapy; CaPSURE, Cancer of the Prostate Strategic Urologic Research Endeavor. 
Matched analysis was undertaken by using greedy propensity scorematching techniques designed to optimize the closeness of matching, with minimal wasted occurrences. ${ }^{25}$ This algorithm matches patient cases to controls by selecting controls whose propensity scores are within 0.03 of matched patient cases to optimize matching while minimizing wasted patient cases. Each patient receiving ADT was propensity-matched to a patient not receiving ADT, thus creating a subset of the original cohort, composed of patients equally likely to be exposed to ADT. Adjusted HRs with $95 \%$ CI were determined from propensity score-adjusted and propensity score-matched analyses. All analyses were performed with SAS 9.1 (SAS Institute, Cary, NC).

\section{RESULTS}

Of the 13,887 men who were enrolled on the CaPSURE database as of $2010,7,248$ met the specified inclusion criteria. Figure 1 illustrates the selection of study patients and exclusion criteria. Table 1 provides an assessment of baseline demographic and clinical parameters of study patients. Overall, 1,572 men (21.7\%) received ADT at some point during their treatment; 5,170 men $(71.3 \%)$ received local treatment alone, 485 men (6.7\%) received local treatment plus ADT, 1,087 men (15\%) received primary ADT, and 506 men (7.0\%) received WW/AS. The mean durations of follow-up were 51.3, 57, 52.6, and 47.6 months in the local treatment alone, local treatment plus ADT, primary ADT, and WW/AS groups, respectively.

Men undergoing different treatments had substantially different clinical characteristics in both cancer risk and comorbidity. In particular, patients who received ADT, either as primary therapy or in combination with local therapy, were older, had more comorbidities, and had greater cancer risk compared with those who received local therapy alone. Although men who received WW/AS were older and had more comorbidity, their cancer risks were closer to patients who received local therapy alone (Table 1 ).

Overall, $976(13.5 \%)$ of men in the data set died as a result of any cause: 103 men (1.4\%) died as a result of prostate cancer, 195 men

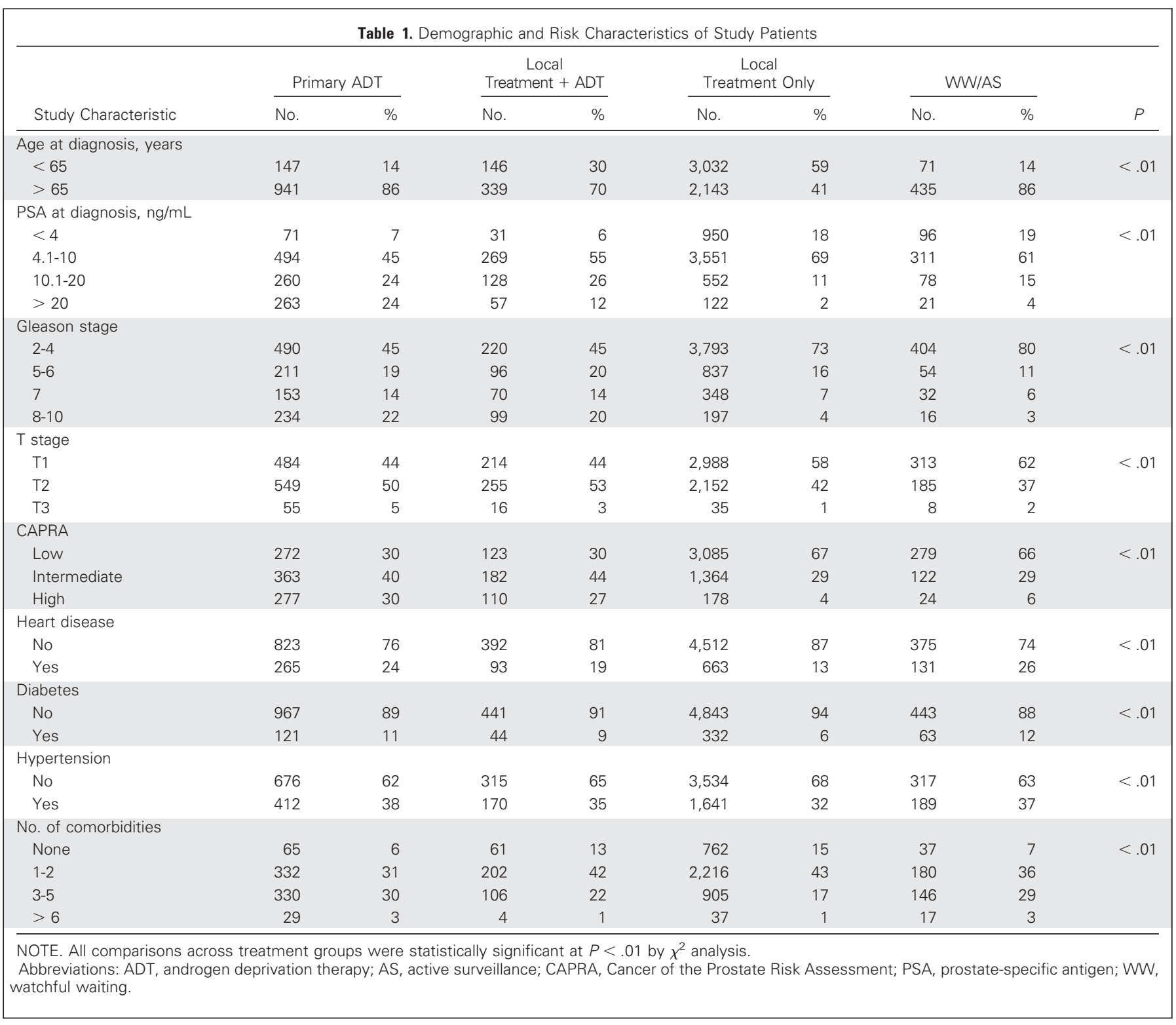




\begin{tabular}{|c|c|c|c|c|c|}
\hline \multirow[b]{2}{*}{ Treatment } & \multicolumn{5}{|c|}{ Cause of Mortality } \\
\hline & Alive & Prostate Cancer Specific & Cardiovascular & Other & Total \\
\hline \multicolumn{6}{|l|}{ Primary ADT } \\
\hline No. & 735 & 52 & 73 & 227 & 1,087 \\
\hline Row \% & 67.62 & 4.78 & 6.72 & 20.88 & 100 \\
\hline Column \% & 11.72 & 50.49 & 37.44 & 33.48 & 15.00 \\
\hline \multicolumn{6}{|l|}{ Local + ADT } \\
\hline No. & 391 & 26 & 16 & 52 & 485 \\
\hline Row \% & 80.62 & 5.36 & 3.30 & 10.72 & 100 \\
\hline Column \% & 6.23 & 25.24 & 8.21 & 7.67 & 6.69 \\
\hline \multicolumn{6}{|l|}{ Local alone } \\
\hline No. & 4,758 & 17 & 75 & 320 & 5,170 \\
\hline Row \% & 92.03 & 0.33 & 1.45 & 6.19 & 100 \\
\hline Column \% & 75.86 & 16.50 & 38.46 & 47.20 & 71.33 \\
\hline \multicolumn{6}{|c|}{ Watchful waiting/active surveillance } \\
\hline No. & 388 & 8 & 31 & 79 & 506 \\
\hline Row \% & 76.68 & 1.58 & 6.13 & 15.61 & 100 \\
\hline Column \% & 6.19 & 7.77 & 15.90 & 11.65 & 6.98 \\
\hline \multicolumn{6}{|l|}{ Total } \\
\hline No. & 6,272 & 103 & 195 & 678 & 7,248 \\
\hline Row \% & 86.53 & 1.42 & 2.69 & 9.35 & 100 \\
\hline Column \% & 100 & 100 & 100 & 100 & 100 \\
\hline
\end{tabular}

Abbreviation: ADT, androgen deprivation therapy.

(2.7\%) died as a result of cardiovascular disease, and 678 men (9.4\%) died as a result of other causes (Table 2). When we compared causes of mortality by type of treatment received, we found that those who were treated with ADT, either as primary treatment or in combination with local therapy, had a higher PCSM than patients treated with local therapy alone, reflecting higher-risk disease. In addition, they had higher CVM. Patients treated with WW/AS also had a greater PCSM and CVM than those treated with local therapy alone (Table 2).

In competing-risks analysis, treatment with ADT, either as primary therapy or in combination with local treatment, was associated with an increased risk of PCSM compared with local treatment alone. Patients in the WW/AS arm also had an increased risk of PCSM compared with patients treated with local therapy alone (Table 3). With respect to CVM, there was an almost two-fold increased risk for those patients treated with primary ADT compared with local therapy alone (HR, 1.94; 95\% CI, 1.29 to 2.97), but there was no increased risk for those patients treated with a combination of local therapy and ADT (HR, 1.36; 95\% CI, 0.65 to 1.98). However, the patients in the WW/AS group had a greater than two-fold increased risk of CVM (HR, 2.46; 95\% CI, 1.53 to 3.95) compared with patients treated with local therapy alone (Table 3 ).

In the propensity-adjusted multivariate analysis, intermediate and higher probability of receiving ADT was associated with an increased mortality for each end point of interest (Appendix Table A1, online only). The propensity-matching algorithm produced a subset of 1,391 patients. Results from this analysis indicated that exposure to ADT was associated with an increase in all-cause mortality (HR, 1.25; 95\% CI, 1.03 to 1.5$)$. However, there was no statistically significant difference in PCSM (HR, 1.53; 95\% CI, 0.87 to 2.69) or CVM (HR, $1.12 ; 95 \% \mathrm{CI}, 0.73$ to 1.67 ) between patients matched by likelihood to receive ADT. Moreover, the strength of any association was significantly lower compared with our nonmatched analysis (Table 4). A

\begin{tabular}{|c|c|c|c|c|c|c|c|c|}
\hline \multirow[b]{3}{*}{ Treatment Course } & \multicolumn{8}{|c|}{ Mortality } \\
\hline & \multicolumn{2}{|c|}{ Overall* $^{*}$} & \multicolumn{2}{|c|}{ Prostate Cancer Specific† } & \multicolumn{2}{|c|}{ Cardiovascular $\ddagger$} & \multicolumn{2}{|c|}{ Other§ } \\
\hline & $\mathrm{HR}$ & $95 \% \mathrm{Cl}$ & HR & $95 \% \mathrm{Cl}$ & HR & $95 \% \mathrm{Cl}$ & $\mathrm{HR}$ & $95 \% \mathrm{Cl}$ \\
\hline Local only & \multicolumn{2}{|c|}{ Ref } & \multicolumn{2}{|r|}{ Ref } & \multicolumn{2}{|c|}{ Ref } & \multicolumn{2}{|c|}{ Ref } \\
\hline ADT only & 1.55 & 1.26 to 1.9 & 6.31 & 3.21 to 12.4 & 1.94 & 1.29 to 2.97 & 1.39 & 1.12 to 1.73 \\
\hline Local + ADT & 1.04 & 0.79 to 1.36 & 6.44 & 3.37 to 12.3 & 1.36 & 0.65 to 1.98 & 0.75 & 0.55 to 1.01 \\
\hline WW/AS & 1.7 & 1.34 to 2.24 & 5.27 & 2.16 to 12.86 & 2.46 & 1.53 to 3.95 & 1.33 & 1.01 to 1.75 \\
\hline \multicolumn{9}{|c|}{$\begin{array}{l}\text { NOTE. For each mortality end point, } \mathrm{HR} \text { is given with } 95 \% \text { Cls for each treatment group relative to local therapy only. } \\
\text { Abbreviations: ADT, androgen deprivation therapy; AS, active surveillance; HR, hazard ratio; Ref, reference; WW, watchful waiting. } \\
{ }^{*} P \text { value significant for treatment, Gleason score, age, pretreatment prostate-specific antigen, clinical stage, number of comorbidities, diabetes, and cardiac disease. } \\
\dagger P \text { value significant for treatment, age, clinical stage, and prostate-specific antigen. } \\
\ddagger P \text { value significant for treatment, age, clinical stage, prostate-specific antigen, and cardiac disease. } \\
\S P \text { value significant for treatment, age, Gleason score, prostate-specific antigen, and number of comorbidities. }\end{array}$} \\
\hline
\end{tabular}




\begin{tabular}{|c|c|c|c|c|c|c|c|c|}
\hline \multirow[b]{3}{*}{ ADT Status } & \multicolumn{8}{|c|}{ Mortality } \\
\hline & \multicolumn{2}{|c|}{ Overall } & \multicolumn{2}{|c|}{ Prostate Cancer Specific } & \multicolumn{2}{|c|}{ Cardiovascular } & \multicolumn{2}{|c|}{ Other } \\
\hline & $\mathrm{HR}$ & $95 \% \mathrm{Cl}$ & $\mathrm{HR}$ & $95 \% \mathrm{Cl}$ & $\mathrm{HR}$ & $95 \% \mathrm{Cl}$ & $\mathrm{HR}$ & $95 \% \mathrm{Cl}$ \\
\hline$\overline{A D T}$ negative & \multicolumn{2}{|c|}{ Ref } & \multicolumn{2}{|c|}{ Ref } & \multicolumn{2}{|c|}{ Ref } & \multicolumn{2}{|c|}{ Ref } \\
\hline ADT positive & 1.25 & 1.03 to 1.5 & 1.53 & 0.87 to 2.69 & 1.12 & 0.73 to 1.67 & 1.24 & 0.9 to 1.56 \\
\hline
\end{tabular}

sensitivity analysis, which included patients treated with secondary ADT for less than 6 months, showed similar CVM outcomes as the initial analysis (Appendix Tables A2 and A3, online only). Among patients included in the initial analysis, $31 \%$ were missing comorbidity data. A sensitivity analysis that excluded these patients failed to show any significant differences (data not shown).

\section{DISCUSSION}

By using a large cohort of men in a national cancer registry, this analysis demonstrated that patients treated by WW/AS had an even higher risk of CVM than patients treated with primary ADT. Given that the baseline demographics and comorbidity status of the WW/AS and primary ADT groups were similar, this suggests that unmeasured factors affecting treatment selection may confound the association between ADT and cardiovascular risk. In an attempt to control for these factors, a propensity-matched analysis was performed, which failed to demonstrate an increased risk of CVM in those patients who received ADT.

A previously published observational study of 73,196 Medicare enrollees with localized prostate cancer assessed whether treatment with GnRH agonists or orchiectomy was associated with diabetes or cardiovascular events. ${ }^{10}$ The authors found that GnRH agonist use was associated with an increased risk of diabetes $(\mathrm{HR}, 1.44 ; P<.001)$, coronary heart disease (HR, $1.11 ; P=.03)$, myocardial infarction $(\mathrm{HR}, 1.11 ; P=.03)$, and sudden cardiac death (HR, 1.16; $P=.004)$. However, men treated with orchiectomy only had an increased risk of diabetes ( $\mathrm{HR}, 1.34 ; P<.001)$ and had no increased risk of the abovementioned cardiovascular events. Another study examining 22,816 men with prostate cancer who were identified retrospectively from a population-based registry assessed the affect of ADT on subsequent cardiovascular morbidity. ${ }^{26}$ Within this cohort, $21 \%$ of patients received ADT at some point during treatment. The authors found that those who received ADT for at least 1 year had a 20\% higher risk of serious cardiovascular morbidity compared with similar men who did not receive $\mathrm{ADT}$.

Finally, a study by Tsai et $\mathrm{al}^{12}{ }^{12}$ which also used the CaPSURE database, described an increased risk of CVM with ADT use. The authors assessed 3,262 patients treated with radical prostatectomy and 1,630 patients treated with external-beam radiation therapy, brachytherapy or cryoablation, of which 1,015 of the total cohort had been treated with neoadjuvant or adjuvant ADT. They compared the use of ADT with time to death as a result of cardiovascular causes between ADT users and nonusers. Among the 266 men undergoing neoadjuvant $\mathrm{ADT}$ before prostatectomy only $(8.1 \%$ of the patients who underwent prostatectomy), the authors reported a significant association between ADT use and CVM (HR, 2.6; 95\% CI, 1.4 to $4.7 ; P=.002)$. There was no similar association between ADT use and CVM in the radiation therapy group.

There is concern, however, in the above-mentioned studies that unmeasured factors maybe confounding the relationship between ADT use and CVM. In the study by Tsai et al, ${ }^{12}$ the analysis for cardiovascular risk adjusted only for age and the presence of coronary disease and diabetes. Furthermore, although the authors saw an increased CVM in men using ADT in the radical prostatectomy group, they did not see this association in the nonsurgical group despite a similar exposure to ADT ( $4.1 v 4.3$ months, respectively). Assessing CVM rather than PCSM end points can be more challenging when using administrative data or data from registries intended to study prostate cancer. Although CaPSURE included comorbidity checklists for diabetes and congestive heart disease, for example, it did not include hemoglobin A1c levels, heart failure severity classifications, or other important indicators of cardiovascular disease risk.

Other reports corroborate findings of the current analysis. A study that used administrative databases in Ontario, Canada, compared 19,079 men who used ADT to 19,079 men who did not to assess the development of acute myocardial infarction, sudden cardiac death, and diabetes. ${ }^{16}$ These men were matched one-to-one on age, prior cancer treatment, and year of diagnosis, and they were propensity-matched on comorbidities, medications, cardiovascular risk factors, prior fractures, and socioeconomic variables. The analysis did not identify an association between ADT use and acute myocardial infarction (HR, $0.91 ; 95 \% \mathrm{CI}, 0.8$ to 1 ) or sudden cardiac death (HR, 0.96 ; $95 \%$ CI, 0.8 to 1.1 ).

Two secondary analyses of large, randomized trials have also supported the findings of the present study. One randomized, phase III trial examining 945 men compared the effects of radiation therapy and adjuvant ADT to radiation therapy alone. After 9 years of followup, CVM for men receiving adjuvant ADT was $8.4 \%$ compared with $11.4 \%$ for men treated without adjuvant ADT, and no significant difference was appreciated between the two arms. ${ }^{18}$ Another recent study analyzing 1,554 men who were randomly assigned to radiation therapy and 4 months or 28 months of adjuvant ADT found no association between the duration of ADT and CVM. ${ }^{17}$ The major advantage of these trials is their ability to control, through random assignment, for both known and unknown factors that may confound the association between ADT and CVM; thus, these findings may be more reliable.

Our study had several strengths, including a large data set of 7,248 men, of whom 195 had cardiovascular events. Another advantage was the addition of a propensity-adjusted and matched analysis to elucidate the effect of ADT on CVM. The study also has limitations. 
Although we were unable to see a significant association between ADT and CVM in the matched analysis, the CIs around our risk estimate ranged from a small protective effect to an almost two-fold increase in risk, suggesting a possible lack of power to identify this association. No a priori sample size calculation was performed, because this study was limited to the patients and events within the CaPSURE database.

Second, death certificates were used to determine the cause of mortality. Previous studies have shown that death certificates can be unreliable, particularly when reporting mortality related to cardiovascular causes. $^{27-29}$ Therefore, there is a possibility of misclassification of CVM as an end point, which may be reflected by the increase in all-cause mortality in ADT users compared with nonusers. However, this increase in overall mortality may be due to many other reasons, such as sequelae of skeletal complications that are unrelated to cardiovascular effects of treatment. Third, evaluation of CVM end points in a registry intended to study prostate cancer can be challenging, because CaPSURE does not include all the important indicators of cardiovascular disease risk. Therefore, this study may also be affected by potential unmeasured confounders, which may affect the observed results. Attempts to minimize this effect were made by using a propensity-matched algorithm to compare ADT users to nonusers.

Another limitation and potential bias was that $31 \%$ of the cohort were missing comorbidity data. However, these patients were equally distributed among the treatment groups, and $25 \%$ to $30 \%$ of patients in each treatment group were missing comorbidity data. These patients were included in the analysis; however, a sensitivity analysis in which these patients were excluded failed to show any significant changes in the risk estimates. In addition, there were concerns regarding the reliability of data pertaining to duration of ADT use. As a result, a distinction between patients on continuous versus intermittent ADT could not be made. Finally, CaPSURE did not differentiate between men on AS and those on WW. These limitations are common to observational studies that use large disease registries.

When studying a large cohort of men with propensity analysis, we were unable to identify an association between ADT use and CVM, which suggests that previous studies that found an association may have been confounded by unmeasured variables that affected both treatment selection and various non-prostate cancer-specific outcomes, including CVM. However, this information does not preclude the need for cardiovascular risk assessment in men being treated with ADT as well as a careful and complete discussion of the need for and risks and benefits of ADT in those being considered for such therapy.

\section{AUTHORS' DISCLOSURES OF POTENTIAL CONFLICTS} OF INTEREST

Although all authors completed the disclosure declaration, the following author(s) indicated a financial or other interest that is relevant to the subject matter under consideration in this article. Certain relationships marked with a " $U$ " are those for which no compensation was received; those relationships marked with a " $C$ " were compensated. For a detailed description of the disclosure categories, or for more information about ASCO's conflict of interest policy, please refer to the Author Disclosure Declaration and the Disclosures of Potential Conflicts of Interest section in Information for Contributors.

Employment or Leadership Position: None Consultant or Advisory Role: None Stock Ownership: None Honoraria: Matthew R. Cooperberg, Abbott Laboratories, Takeda Pharmaceuticals; Peter R. Carroll, Takeda Pharmaceuticals Research Funding: None Expert Testimony: None Other Remuneration: None

\section{AUTHOR CONTRIBUTIONS}

Conception and design: Matthew R. Cooperberg, Peter R. Carroll Financial support: Peter R. Carroll

Administrative support: Peter R. Carroll

Provision of study materials or patients: Peter R. Carroll

Collection and assembly of data: Matthew R. Cooperberg, Natalia

Sadetsky, Peter R. Carroll

Data analysis and interpretation: All authors

Manuscript writing: All authors

Final approval of manuscript: All authors

\section{REFERENCES}

1. Jemal $A$, Siegel $R, X u$ J, et al: Cancer statistics, 2010. CA Cancer J Clin 60:277-300, 2010

2. Greenlee RT, Murray T, Bolden $S$, et al: Cancer statistics, 2000. CA Cancer J Clin 50:7-33, 2000

3. Quon H, Loblaw DA: Androgen deprivation therapy for prostate cancer-review of indications in 2010. Curr Oncol 17:S38-S44, 2010 (suppl 2)

4. Bolla $M$, Collette L, Blank L, et al: Long-term results with immediate androgen suppression and external irradiation in patients with locally advanced prostate cancer (an EORTC study): A phase III randomised trial. Lancet 360:103-106, 2002

5. Heidenreich A, Bellmunt J, Bolla M, et al: EAU Guidelines on prostate cancer. Part 1: Screening, diagnosis, and treatment of clinically localised disease. Eur Urol 59:61-71, 2011

6. Boukaram C, Hannoun-Levi JM: Management of prostate cancer recurrence after definitive radiation therapy. Cancer Treat Rev 36:91100, 2010

7. Meng MV GG, Sadetsky N, Mehta SS, et al: Contemporary patterns of androgen deprivation therapy use for newly diagnosed prostate cancer. Urology 60:7-11, 2002 (suppl 1)

8. Cooperberg MR, Grossfeld GD, Lubeck DP, et al: National practice patterns and time trends in androgen ablation for localized prostate cancer. J Natl Cancer Inst 95:981-989, 2003

9. D'Amico AV, Denham JW, Crook J, et al: Influence of androgen suppression therapy for prostate cancer on the frequency and timing of fatal myocardial infarctions. J Clin Oncol 25:2420-2425, 2007

10. Keating NL, O'Malley AJ, Smith MR: Diabetes and cardiovascular disease during androgen deprivation therapy for prostate cancer. J Clin Oncol 24: 4448-4456, 2006

11. Nanda $A$, Chen $M H$, Braccioforte $M H$, et al: Hormonal therapy use for prostate cancer and mortality in men with coronary artery disease-induced congestive heart failure or myocardial infarction. JAMA 302:866-873, 2009

12. Tsai HK, D'Amico AV, Sadetsky $N$, et al: Androgen deprivation therapy for localized prostate cancer and the risk of cardiovascular mortality. J Natl Cancer Inst 99:1516-1524, 2007

13. Smith MR, Finkelstein JS, McGovern FJ, et al: Changes in body composition during androgen deprivation therapy for prostate cancer. J Clin Endocrinol Metab 87:599-603, 2002
14. Smith MR: Changes in fat and lean body mass during androgen-deprivation therapy for prostate cancer. Urology 63:742-745, 2004

15. Smith MR, Lee $H$, Nathan DM: Insulin sensitivity during combined androgen blockade for prostate cancer. J Clin Endocrinol Metab 91:1305-1308, 2006

16. Alibhai SM, Duong-Hua M, Sutradhar R, et al: Impact of androgen deprivation therapy on cardiovascular disease and diabetes. J Clin Oncol 27:34523458, 2009

17. Efstathiou JA, Bae K, Shipley WU, et al: Cardiovascular mortality and duration of androgen deprivation for locally advanced prostate cancer: Analysis of RTOG 92-02. Eur Urol 54:816-823, 2008

18. Efstathiou JA, Bae K, Shipley WU, et al: Cardiovascular mortality after androgen deprivation therapy for locally advanced prostate cancer: RTOG 85-31. J Clin Oncol 27:92-99, 2009

19. Kim HS, Freedland SJ: Androgen deprivation therapy in prostate cancer: Anticipated side-effects and their management. Curr Opin Support Palliat Care 4:147-152, 2010

20. Cooperberg MR, Broering JM, Litwin MS, et al: The contemporary management of prostate cancer in the United States: Lessons from the cancer of the prostate strategic urologic research endeavor 
(CapSURE), a national disease registry. J Urol 171: 1393-1401, 2004

21. Lubeck DP, Litwin MS, Henning JM, et al: The CaPSURE database: A methodology for clinical practice and research in prostate cancer-CaPSURE Research Panel: Cancer of the Prostate Strategic Urologic Research Endeavor. Urology 48:773-777, 1996

22. Allison PD: Survival Analysis Using the SAS system: A Practical Guide. SAS Institute, Cary, NC, 1995

23. Cooperberg MR, Broering JM, Carroll PR: Risk assessment for prostate cancer metastasis and mortality at the time of diagnosis. J Natl Cancer Inst 101:878-887, 2009

24. Rubin DB: Estimating causal effects from large data sets using propensity scores. Ann Intern Med 127:757-763, 1997

25. Parsons LS: Reducing bias in a propensity score matched-pair sample using greedy matching techniques. SUGI 26, 2001 (abstr 214-226)

26. Saigal CS, Gore JL, Krupski TL, et al: Androgen deprivation therapy increases cardiovascular morbidity in men with prostate cancer. Cancer 110: 1493-1500, 2007
27. Beer V, Schick MT, Minder CE: Mortality analysis: When is single evaluation of the basic cause of death allowable, when should multicausality be assessed? [in German] Soz Praventivmed 35:17-23, 1990

28. Hunt LW Jr, Silverstein MD, Reed CE, et al: Accuracy of the death certificate in a populationbased study of asthmatic patients. JAMA 269:19471952, 1993

29. Mühlhauser I, Sawicki $P$, Blank $M$, et al: Reliability of causes of death in person with type 1 diabetes. Diabetologia 45:1490-1497, 2002

\section{Help Your Patients Understand Advanced Cancer Care Planning}

ASCO's Advanced Cancer Care Planning booklet is designed to help people with advanced cancer and their families and caregivers understand the diagnosis and treatment options for advanced cancer, discuss these options for care throughout the course of the illness, and find support. Download the booklet in English and Spanish at www.cancer.net/advancedcancer or order copies for your practice at www.cancer.net/estore. 